\title{
Sciences, technologie, société
}

L'enseignement des sciences aux États-Unis

\section{Edward D. Britton}

Traducteur : Sylvie Bronner

\section{OpenEdition}

\section{Journals}

Édition électronique

URL : http://journals.openedition.org/ries/3347

DOI : $10.4000 /$ ries.3347

ISSN : 2261-4265

\section{Éditeur}

Centre international d'études pédagogiques

\section{Édition imprimée}

Date de publication : 1 juin 1997

Pagination : 61-66

ISSN : 1254-4590

\section{Référence électronique}

Edward D. Britton, «Sciences, technologie, société », Revue internationale d'éducation de Sèvres [En ligne], 14 | 1997, mis en ligne le 30 juillet 2013, consulté le 22 avril 2019. URL : http:// journals.openedition.org/ries/3347 ; DOI : 10.4000/ries.3347

Ce document a été généré automatiquement le 22 avril 2019

(c) Tous droits réservés 


\title{
Sciences, technologie, société
}

\author{
L'enseignement des sciences aux États-Unis
}

\author{
Edward D. Britton
}

Traduction : Sylvie Bronner

1 "ChemCom» (Chemistry in the Community), la chimie dans la vie quotidienne, est un des meilleurs exemples de la façon dont le programme scientifique dans les écoles américaines donne une importance croissante aux applications scientifiques en technologie et dans la société. Ce programme a été lancé en 1988 par la Société américaine de chimie ${ }^{2}$. Ce nouveau programme était l'une des huit innovations étudiées durant la période pendant laquelle les États-Unis ont pris part au projet de l'OCDE sur des études de cas concernant les innovations en sciences, mathématiques et enseignement technologique.

2 Enseigner les connaissances de base des disciplines scientifiques a été et demeure toujours l'objectif essentiel dans la plus grande partie du temps d'enseignement. Cependant, il y a toujours eu des partisans d'un enseignement des sciences faisant apparaître l'utilité des sciences dans la vie quotidienne de l'individu ${ }^{3}$ et dans la société, en général. Durant les deux dernières décennies, la demande s'est accrue pour inclure davantage des sciences appliquées. Un signe qui montre la place grandissante des sciences appliquées dans le débat sur les programmes : désormais, dans les publications il est souvent fait allusion à ce phénomène en utilisant l'acronyme STS pour sciencestechnologie-société.

\section{Le projet STS}

Dans les dix dernières années, on a généralement accepté le fait que les programmes de sciences incluent l'approche STS à l'école. Cette tendance a débuté par la déclaration suivante: alors que le programme de sciences a traditionnellement et largement contribué à la préparation des futurs scientifiques, il doit maintenant amener tous les élèves à la compréhension des sciences. Cet objectif a même été adopté par la Fondation nationale des sciences (NSF), un organisme fédéral qui finance non seulement la 
recherche scientifique fondamentale, mais aussi une grande partie de la recherche nationale sur l'enseignement des sciences. La NSF a contribué pour plus d'un million de dollars au développement de ChemCom. Un corollaire du principe de la science pour tous est que les connaissances scientifiques de base incluent l'approche STS et également des connaissances dans la discipline. En 1996, le prestigieux Conseil national de la recherche publia, pour la première fois dans l'histoire des États- Unis, des normes minimales concernant l'enseignement facultatif des sciences au niveau national ${ }^{4}$. Ce document de deux cent cinquante pages comporte des sections concernant le savoir scientifique traditionnel tel que les sciences physiques et les sciences de la vie. Cependant le livre consacre également autant d'attention aux normes en «sciences et technologie » et en «sciences dans des implications individuelles et sociales».

4 La réponse de la plupart des concepteurs de programmes a été de traiter les sciences appliquées comme un ajout à la connaissance de la discipline; par exemple, de nombreux manuels scolaires scientifiques américains ajoutent quelques paragraphes sur les applications à la fin de chaque thème scientifique important. ChemCom adopte une approche plus radicale sur la discipline ; au lieu de se contenter d'ajouter des éléments de cet ordre, il structure le programme de chimie des high schools en fonction de la démarche STS.

\section{Le contexte impose le contenu}

5 Chacune des huit unités du cours annuel de ChemCom utilise des sujets d'actualité concrets pour mêler des sujets concernant les sciences, la technologie et la société. Des exemples de titres d'unités : « subvenir à nos besoins en eau », « la gestion des ressources chimiques", " comprendre la nourriture ", «la santé : les risques et choix à prendre ». ChemCom est en fait beaucoup plus qu'une approche de la chimie appliquée ; à l'inverse, il met l'accent sur les principales questions touchant à l'environnement et la santé qui ont un ancrage dans la chimie et constituent des créneaux porteurs dans l'activité des élèves dans leur milieu social. Les cours de chimie classiques offrent aux élèves des concepts disciplinaires dans le but de les préparer aux cours universitaires. ChemCom est un programme centré sur des sujets d'actualité qui intègrent des sujets de chimie selon les besoins de la vie quotidienne. Ce qui signifie que les concepts ne sont introduits que parce qu'ils sont nécessaires pour comprendre la chimie.

Les commentaires suivants d'un professeur de ChemCom éclairent les objectifs et les caractéristiques :

«ChemCom contribue à faire des élèves de meilleurs consommateurs tout en leur permettant de comprendre l'importance de la chimie par rapport à la société, au pays et au monde. On insiste fortement sur les capacités d'apprentissage en commun et la prise de décision essentielle pour leur permettre d'entrer dans la vie active. ChemCom les forme à la compréhension de la chimie en liaison avec le monde.»

Pour illustrer le thème de ChemCom, considérons le chapitre sur la qualité de l'eau. Ce chapitre recouvre les thèmes de la chimie classique tels que les types de solutions et les propriétés, les caractéristiques des acides et des bases et la structure moléculaire de l'eau. ChemCom intègre un travail dans les laboratoires traditionnels utilisant des éprouvettes graduées pour mesurer et filtrer les liquides et évaluer la dissolution des produits solubles dans des solvants polaires et non polaires. Toutefois ChemCom comporte du 
travail sur thème et en laboratoire rarement abordé dans les cours de chimie traditionnels : les causes et les effets de la contamination par les métaux lourds, le traitement de l'eau dans les collectivités locales, les procédés d'adoucissement de l'eau et les mesures de la pureté des eaux usées ${ }^{5}$. Globalement ChemCom recouvre beaucoup plus de biochimie, de chimie organique et nucléaire que les cours traditionnels.

8 À travers les huit chapitres, les enseignants retrouvent la plupart des principaux thèmes qui figurent dans un cours de chimie traditionnel. Cependant, la plupart des enseignants découvrent qu'il est possible de traiter cinq ou six chapitres dans l'année. De plus, ChemCom laisse de côté les sujets classiques parce qu'ils n'ont pas de valeur pour expliquer les questions essentielles de la société : atomes et molécules, cinétique, équilibre et énergie en réaction. Beaucoup d'enseignants de ChemCom étant habitués à enseigner la chimie traditionnelle, trouvent déconcertant le fait d'omettre les thèmes traditionnels de ce genre. Ils sont tentés d'escamoter certaines données du programme ChemCom pour avoir le temps d'insérer les thèmes traditionnels éludés par ChemCom. Les enseignants sont pourtant généralement satisfaits de voir avec quel succès leurs élèves s'engagent dans le projet STS, et bien souvent ils envisagent d'utiliser certains thèmes de ChemCom pour rendre leurs cours traditionnels plus attrayants.

Beaucoup d'élèves sont très attirés par cette approche STS. Dans l'étude de cas de l'OCDE, quelques enseignants de ChemCom leur ont soumis un questionnaire alors qu'ils venaient de terminer le cours et ils ont découvert que $100 \%$ pensaient qu'ils avaient étudié la chimie qui leur était utile dans leur vie et que $85 \%$ déclaraient qu'ils suivraient à nouveau Chem Com s'ils devaient choisir un cours de chimie. Les commentaires suivants d'un élève sont caractéristiques :

«J'ai appris beaucoup cette année, de la résolution des équations au recyclage. J’ai profité de ce cours plus que d'aucun autre auparavant. Nous avons abordé tellement de sujets que je reverrai dans la vie de tous les jours, comme la puissance nucléaire et les gaz dans notre atmosphère. Je comprends mieux maintenant le monde qui m'entoure. »

\section{La pédagogie change}

Si ChemCom constitue un changement radical dans le contenu des cours, il nécessite également un changement significatif dans la pédagogie. ChemCom comporte des questions et exercices que les élèves traitent individuellement, comme dans les cours traditionnels. Mais ChemCom contient également de nombreuses activités de résolution de problèmes d'une durée de trente à cinquante minutes qui nécessitent un travail collectif ou par groupes. Mais les activités les plus novatrices appelées "Mises en commun » se situent à la fin de chaque chapitre. Les élèves doivent revoir et faire la synthèse de leurs connaissances pour aborder la question sous l'angle de la société. Cela se fait en classe entière et peut se dérouler sur un ou deux jours ensemble et plusieurs jours de travail préparatoire individuel ou par petits groupes.

11 Par exemple, le point d'aboutissement du chapitre sur l'eau dans ChemCom est la simulation d'un conseil municipal qui tente de décider ce qu'il convient de faire en matière de qualité de l'eau. Les élèves tiennent le rôle de conseillers municipaux, responsables de compagnies, scientifiques, ingénieurs, propriétaires... Chaque catégorie a deux minutes pour faire une présentation devant le conseil. Après la réunion, les élèves doivent construire leur argumentation en rédigeant des lettres aux journaux ou en se 
préparant à des interviews télévisées. De telles activités offrent des défis pédagogiques aux enseignants habitués aux cours traditionnels et exigent des élèves des prises de décisions sophistiquées. En fait, c'est cet aspect de ChemCom qui a incité les chercheurs lors de la troisième étude internationale sur les mathématiques et les sciences à inclure une rubrique " prise de décision » dans le programme TIMSS ${ }^{6}$.

\section{Un cadre pour la science ${ }^{7}$}

Dans les activités de ChemCom, les enseignants doivent faciliter l'apprentissage des élèves en petits ou grands groupes et réduire l'enseignement de type magistral d'un rôle central à un rôle mineur. Les enseignants de chimie classique y voient un défi et la majorité des enseignants de ChemCom sont des enseignants de chimie classique à qui on a demandé d'enseigner ce nouveau cours.

13 Les chercheurs ont constaté que les enseignants qui ne prennent pas part à des stages de formation avant la première utilisation de ChemCom ont tendance à minimiser les stratégies pédagogiques innovantes. La Société américaine de chimie et Kendall/Hunt, l'éditeur du programme, ont tous deux proposé des ateliers de cinq ou dix jours pour la formation des futurs enseignants. Durant les premières années, ces ateliers furent un élément décisif dans l'introduction de ce programme dans les écoles. Les commentaires suivants illustrent le point de vue favorable des enseignants sur la formation :

«Je le recommande particulièrement. On comprend concrètement ce qu'est le programme et comment on peut l'enseigner. Cela a été une expérience très positive et je n'aurais certainement pas démarré aussi bien si je n'avais pas suivi cette formation ».

\section{La place de ChemCom dans l'enseignement secondaire}

Le cours ChemCom était fait à l'origine pour les élèves se destinant à des études supérieures, mais non spécialisées en sciences. Ses concepteurs avaient le sentiment que des élèves qui n'étaient pas destinés à l'université mais qui avaient des capacités intellectuelles, pouvaient aussi en bénéficier. L'objectif de ChemCom n'est donc pas de remplacer les cours de chimie classique mais d'offrir une alternative qui attire des élèves doués qui sinon n'auraient pas choisi un cours de chimie. La Société américaine de chimie prétend que si la société doit s'attaquer à des questions d'actualité sujettes à controverse et qui affectent la qualité de la vie, telles que la pollution de l'eau et la production d'énergie nucléaire, un plus grand nombre de diplômés des high schools a besoin de comprendre la chimie.

15 À côté des aspects novateurs de ChemCom, un autre facteur a sans doute contribué à son succès. Dans les années 1980, la plupart des cinquante États ont augmenté le nombre de cours de sciences que tous les élèves doivent suivre avec succès pour obtenir le diplôme de fin d'études secondaires. Les élèves de high schools doivent maintenant suivre des cours de sciences pendant deux ou trois ans au lieu d'un an auparavant. En conséquence, les établissements se sont montrés désireux de développer des cours de sciences différents pour de nombreux élèves qui auraient eu des difficultés à suivre un cours classique de chimie ou de physique.

Même si ChemCom est un cours de nature différente, il n'est pas destiné aux élèves en difficulté. Cependant beaucoup d'écoles dirigent ces élèves vers le cours ChemCom et les 
enseignants doivent parfois modifier ChemCom pour s'adapter aux capacités de ces élèves. O’Brien déclare ${ }^{8}$ :

«Bien que ChemCom exige un niveau en mathématiques moindre qu'un cours de chimie classique, la complexité conceptuelle des sujets de STS et le processus de prise de décision exigent des capacités mentales et une maturité sociale que l'on attend des élèves se destinant à des études universitaires. ChemCom n'a pas été conçu pour les élèves ayant un niveau de lecture insuffisant ou des problèmes de motivation importants... Ce message n'a pas été transmis avec assez de vigueur aux chefs d'établissement, aux conseillers d'orientation et aux chefs des départements scientifiques et, en conséquence, certains enseignants se retrouvent dans la situation peu enviable d'appliquer un programme mal adapté aux élèves. »

\section{Développement de ChemCom}

La Société américaine de chimie considère ChemCom comme un cours nouveau et attractif qui a, de fait, poussé davantage d'élèves vers la chimie. Jusqu'en 1995, trois cent mille manuels ont déjà été vendus et la Société est en train de préparer une troisième édition. À ce jour, ChemCom a été traduit en japonais, russe et espagnol. Le succès de ChemCom est tel que la Société a repris les caractéristiques essentielles du programme pour élaborer des cours de chimie à l'intention des colleges et des middle schools. En conclusion, voici une autre citation qui montre l'impact de ChemCom sur les élèves :

«En choisissant ChemCom, je comprends l'importance des sciences. J'ai choisi de devenir un scientifique et j'ai réfléchi à des questions importantes qui ne m'apparaissaient pas aussi importantes auparavant. Par exemple, les centrales nucléaires. Je n'avais jamais compris de quoi il s'agissait avant notre discussion de cette année. Même quand je rentre à la maison, je me surprends à essayer de recycler et d'être conscient de l'environnement. Je suis content d'avoir suivi ce cours parce que cela va m'aider à l'université et aussi j'ai appris des choses qui me resteront pour toujours. »

ChemCom ayant ce type d'effet, on peut comprendre que les enseignants de chimie traditionnels aient envie d'essayer.

\section{NOTES}

2. American Chemical Society, ChemCom: Chemistry in the Community, Dubuque (Iowa), Kendal/

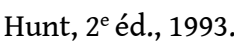

3. M. B. Rowe, J. Montgomery, M. Midling, T. Keating, «ChemCom's Evolution: Development, Spread and Adaptation ", Bold Ventures, vol. 2, Cases Studies of U.S. Innovations in Science Education, S. Raizen et E. Britton (éd.), Dordrecht (Netherlands), Kluwer Academic Publishers, 1996, p. 519-584.

4. National Research Council, National Science Education Standards, Washington, DC, National Academy Press, 1996.

5. D'autres exemples et une description des thèmes de ChemCom sont présentés dans : M. Lynch et E. Britton, «Chemistry in the Community », Sciences and Mathematics Education in the United States: Eight Innovations, Paris OCDE, 1993, p. 59-74. 
6. Third International Mathematics and Science Study, Voir Revue internationale d'éducation de Sèvres $\mathrm{n}$ - 12, décembre 1996, p. 119 et suivantes.

7. Voir «Performance Expectations» (2.2.5), dans D. Robitaille, W. Schmidt, S. Raizen, C. McKnight, E. Britton et C. Nicol, Curriculum Frameworks for Mathematics and Science, TIMMS monographie $n^{\circ}$ 1, Vancouver, Pacific Educational Press, 1993.

8. T. O'Brien, Survey Results from the Summer 1998 ChemCom Resource Teachers Workshop, Binghamton, State University of New York.

\section{RÉSUMÉS}

Le cours de chimie, Chemistry in the Community, n'est pas destiné à des spécialistes de cette discipline mais doit permettre à un plus grand nombre d'élèves de saisir les liens entre sciences, technologie et société. Le succès de cette approche de l'enseignement scientifique a nécessité de la part des enseignants un important effort d'adaptation et de formation.

\section{INDEX}

Index géographique : États-Unis

Mots-clés : chimie, éducation scientifique, études scientifiques, programme d'enseignement, sciences, curriculum

\section{AUTEURS}

\section{EDWARD D. BRITTON}

Associate director, The National Center for Improving Science Education, Washington, ÉtatsUnis. 